D. Revista Humanismo

D 10 y Sociedad

\title{
Las prácticas tutoriales y la formación integral del aprendiz del SENA ${ }^{\Perp}$
}

\author{
Tutorials practical and comprehensive training of SENA Apprentices
}

\author{
Luis Alberto Ospina Ospina, Magíster \\ Mirian Fanny Obando Obando, Magíster \\ Blanca Inés Restrepo Echeverri, Magíster
}

(Aceptado: 4 de diciembre de 2014)

\section{Resumen}

Esta investigación describe y analiza las prácticas tutoriales que son parte de la formación integral del Servicio Nacional de Aprendizaje-SENA en los componentes social, cognitivo y emocional, la cual le permite a esta entidad gubernamental atender las demandas que hoy hacen los empleadores dentro de los parámetros de la responsabilidad social que le fue asignada en la formación del talento humano requerido por el sector productivo en Colombia. Los resultados obtenidos permiten deducir que para mejorar las habilidades de los aprendices en lo social, cognitivo y emocional, se deben reforzar las prácticas tutoriales que hoy desarrollan los instructores de modalidad presencial, para que puedan abordar de manera significativa su rol como Instructor Tutor en la formación integral del aprendiz SENA. Los instructores encuestados reconocen esa dificultad y los aprendices reclaman con vehemencia esa figura que les facilite, no solo la adquisición de conocimientos técnicos, sino también la internalización de valores, actitudes y formas de comportamiento que contribuyan al mejoramiento de sus condiciones sociales y laborales.

Palabras clave: aprendiz, cognitiva, emocional, formación integral, instructor, prácticas tutoriales, social.

\section{Abstract}

This study describes and analyzes the tutorial practices carried out during the integral formation at the National Learning Service, SENA. The social, cognitive and emotional components of SENA allow this governmental institution to meet the current demands of employers, thus fulfilling its social responsibility related with the formation of human talent required by the Colombian productive sector. According to our results, improving trainees education in social, cognitive and emotional skills require to strengthen tutorial presential practices, so instructors can meaningfully address their role as tutors during the integral training of SENA trainees. The respondent instructors recognize this difficulty

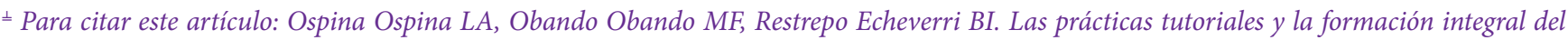
aprendiz del SENA. RHS. Revista. Humanismo. Soc, Volumen 2 (2): 24-37.

*Autorpara correspondencia: Blanca Inés Restrepo Echeverri. Corporación Universitaria Remington, Correo electrónico: brestrepo@uniremington. edu.co.
}

Revista. Humanismo.Soc. 2014; Volumen 2 (2): 24-37. 
and apprentices claim vehemently the figure of a tutor that facilitates not only the acquisition of technical knowledge but also the internalization of values, attitudes and behaviors that contribute to their social and work conditions.

Key words: apprentice, cognitive, comprehensive training, emotional, instructor, tutorials practices, social.

\section{Introducción}

Se hace necesario que el SENA implemente estrategias que le permitan mejorar desde la integralidad de la formación profesional, los componentes cognitivo, social y emocional de los aprendices que la sociedad le ha entregado, lo que le permitirá fortalecer su imagen ante los empresarios. Desde esta perspectiva se planteó el problema que dio vida a la investigación: ¿Cuáles prácticas del instructor tutor promueven la formación integral en los aprendices de Tecnología en Gestión de Mercados del Centro de Comercio del SENA - Regional Antioquia?

El objetivo general de la investigación fue el análisis de las prácticas del instructor tutor que promueven la formación integral del aprendiz de Tecnología en Gestión de Mercados del Centro de Comercio del SENA Regional Antioquia, desde los componentes cognitivo, social y emocional; adicionalmente se establecieron como objetivos específicos, identificar y clasificar las prácticas del Instructor Tutor que promueven habilidades en los aprendices desde dichos componentes y analizarlas a la luz de los aportes teóricos y conceptuales que sustentan la importancia de la interrelación de esas habilidades en la Formación Integral.

\section{Materiales y métodos}

Para delimitar el alcance de la investigación, se asociaron a cada componente las habilidades objeto del estudio: Cognitivo (Indagación, Análisis, Argumentación), Social (Interacción, Trato, Acompañamiento) y Emocional (Tolerancia, Empatía).

\section{Contexto de la formación integral}

Ámbito internacional. El concepto de formación integral hace referencia a la orientación metodológica que promueve el crecimiento humano, a través de un proceso que implica una visión multidimensional del ser humano.
La formación integral favorece el proceso de socialización del estudiante, contribuye a su desarrollo moral y al fortalecimiento de su pensamiento crítico. En ese sentido, va más allá de la sola capacitación, ya que atiende cabalmente al educando como un ser que debe adquirir una preparación para su desempeño en la vida, pero que ante todo tiene derechos y compromisos con una realidad y con el otro que en ella habita, con el fin de contribuir a mejorar sus propias condiciones de vida y las de la sociedad. En este marco, la Comisión Internacional sobre la Educación del Siglo XXI creada por la Unesco, plantea la necesidad de formar una nueva cultura ciudadana que permita a los individuos participar activamente en el desarrollo de la sociedad de su entorno, así como estimular la cooperación entre los seres humanos sin distinción de raza, religión o lengua. En la misma forma, la educación debe fundamentar conocimientos, valores, actitudes y aptitudes favorables a la construcción de una cultura de paz y democracia (Declaración de la 44 Reunión de la Conferencia Internacional de Educación. Ginebra, 1994).

Así, la formación como proceso a lo largo de la vida, de acuerdo con el informe Delors sobre Educación para el Siglo XXI, nos induce a pensar en la necesidad de plantear desde la escuela un nuevo proyecto educativo centrado en el ser humano y no solo en el desarrollo del conocimiento; una propuesta que se inserte en las demandas que la sociedad actual propone, como alternativa a la solución de los principales problemas económicos, políticos, sociales y culturales.

Ámbito nacional. En la educación colombiana la formación profesional es un servicio que complementa la educación básica y puede articularse con diferentes niveles de la educación superior, desarrollando las competencias requeridas para que las personas puedan desempeñarse en diversos sectores productivos.

Para Monroy (1994) la formación integral es aquel proceso a través del cual el hombre desarrollaba todas 
sus facultades en función de su propia espiritualidad $y$ en función del entorno social y natural con el que estaba conectado (Monroy, 1994: pág 148).

La formación integral en el ámbito universitario colombiano, marco de reflexión propuesto por el Ministerio de Educación Nacional (2009) plantea:

- Lograr que la escuela sea efectivamente educadora y no desatienda la diversidad de perspectivas de emplazamiento de los conocimientos, adoptando como guía y sustento la "formación integral" de los individuos.

- Preparar a la sociedad colombiana en su conjunto para la inserción en el mundo global, a partir del desarrollo del talento creador de los individuos en su proceso de involucramiento con diversas estrategias de conocimiento y manejo de la información.

En el SENA. La Formación Profesional Integral hace parte de la Misión de la entidad, la cual ejecuta a través de sus 116 centros de formación distribuidos en 33 regionales, uno de ellos el Centro de Comercio del SENA Regional Antioquia, en donde se llevó a cabo esta investigación.

El Consejo Directivo Nacional del SENA expidió el Acuerdo 12 de 1985, por medio del cual se establecen los lineamientos fundamentales de la política técnicopedagógica y se fijan las directrices para su gestión con miras a lograr y conservar la Unidad Técnica en la entidad. En este acuerdo la formación profesional integral se define como:

La Formación Profesional Integral es un proceso mediante el cual la persona adquiere y desarrolla de manera permanente conocimientos, destrezas y aptitudes e identifica, genera y asume valores y actitudes para su realización humana y su participación activa en el trabajo productivo y en la toma de decisiones sociales.

En este contexto, la Ley 119 del 9 de febrero de 1994 reestructura el Servicio Nacional de Aprendizaje SENA, derogando el Decreto 2149 de 1992 y dicta otras disposiciones. Esta nueva normativa establece la misión del SENA así: ...está encargado de cumplir la función que corresponde al Estado de invertir en el desarrollo social y técnico de los trabajadores colombianos, ofreciendo y ejecutando la formación profesional integral, para la incorporación y el desarrollo de las personas en actividades productivas que contribuyan al desarrollo social, económico y tecnológico del país.

Por otra parte, el Consejo Directivo Nacional del SENA en ejercicio de sus facultades legales y estatutarias, en especial de las que le confiere la Ley 119 de 1994 expide el Acuerdo 00008 de 1997 conocido como Estatuto de la Formación Profesional Integral, plantea que el mundo de la vida está conformado por los contextos productivo y social. Así entonces, el marco social hace referencia a las interacciones de carácter intra e interpersonal, orientadas a la construcción de la persona y de la sociedad y al desarrollo humano integral en los niveles personal y social, así como en la dimensión ambiental. En efecto, la formación profesional incorpora el desarrollo de valores, de habilidades relacionales y de comunicación para contribuir al fortalecimiento de la convivencia y la participación en una sociedad en conflicto, con diferencias étnicas, económicas y sociales.

Durante el periodo comprendido entre 2002 - 2010, el SENA encamina su estrategia hacia el enfoque por competencias; en 2004, la entidad define que el rol del Instructor tutor como responsable del proceso de acompañamiento, no solo es de tipo académico sino también de tipo personal, en aras de que le permita al aprendiz solucionar problemas escolares, desarrollar hábitos de estudio y trabajo, predisposición a la reflexión y a la convivencia social. Igualmente, se implementa el aprendizaje por proyectos como estrategia pedagógica, basado en las cuatro fuentes de conocimiento: instructor tutor - orientador - facilitador, el entorno, las TIC y el trabajo colaborativo, con el propósito de formar aprendices librepensadores, críticos, autónomos, líderes, solidarios y emprendedores.

Aparece entonces el nuevo rol del Instructor tutor, quien retroalimenta y evalúa al aprendiz durante su proceso formativo, haciendo uso de distintas técnicas didácticas activas, bajo la estrategia del aprendizaje por proyectos, la cual le permite contribuir en su propio aprendizaje. 
Cabe resaltar que el aprendizaje por proyectos es una metodología de formación que facilita el desarrollo del aprendizaje basado en problemas, permitiendo desarrollar en el aprendiz un proceso didáctico propio con una mayor responsabilidad y un papel activo en su formación, a partir de la planeación, análisis y desarrollo de actividades concretas para proponer soluciones prácticas que contribuyan a la mejora y al desarrollo de su entorno. Es decir, es una estrategia que reconoce como el aprendizaje significativo conduce al aprendiz a un proceso inherente de conocimiento.

De otro lado, en marzo de 2012 el SENA entrega a la comunidad educativa su Modelo Pedagógico Institucional como una respuesta desde una perspectiva humanística con pertinencia y calidad frente a los retos que para la formación profesional integral de cara al Siglo XXI. Plantea un mundo caracterizado por la globalización, el avance tecnológico, la sociedad del conocimiento, la complejidad y el cambio acelerado y permanente, factores a su vez, ligados a realidades tales como la desigualdad, la pobreza, la exclusión, la violencia, la corrupción, el relativismo en materia de principios y valores éticos y el deterioro ambiental.

\section{Contexto de la tutoría}

Ámbito internacional. El término tutor viene del latín tutor, -oris, o sea, persona encargada de orientar a los alumnos de un curso o asignatura; defensor, protector o director en cualquier línea. Igualmente, la palabra tutor viene del verbo tuerí, es decir, observar, vigilar, proteger. De ahí se derivan palabras como Intuir que significa observar íntimamente.

La Unesco (1998), en la Declaración Mundial sobre la Educación Superior en el Siglo XXI, plantea en su artículo 10: "El personal y los estudiantes, principales protagonistas de la Educación Superior" y que por lo tanto, "habría que desarrollar los servicios de orientación para facilitar el paso de los estudiantes secundarios a la enseñanza superior (...) y para tener en cuenta las necesidades de categorías cada vez más diversificadas de educandos (...)”.

Ámbito nacional. En Colombia el desarrollo de la tutoría no ha tenido una definición y una trayectoria significativa; todo lo que se dice de ella es prestado y acomodado a las filosofías de los proyectos educativos de otros países. Se carece de una reflexión seria en torno a la práctica tutorial, al significado de su concepto, a su perfil y por ende a su aplicación.

En Colombia la tutoría fue aplicada en la década del 70 a la educación a distancia como una herramienta para la asesoría académica, así como facilitación, coordinación y monitorias, lo cual promovía la interacción entre estudiantes, mantenían su motivación, se les brindaba información y se conservaba así la relación con la institución. En este contexto, el Instituto Colombiano para el Fomento de la Educación Superior - Icfes, en 1981 presentó las directrices para la labor y objetivos de la tutoría y definió que los tipos de esta son la presencial, telefónica, escrita y radial, las cuales se ajustaban en su momento a los requerimientos del sistema educativo. Por su parte, la Ley de Educación Superior (Ley 30 de 1992) mediante la cual se organizó el servicio público de la educación superior, no hace mención frente a la tutoría, pero sí estableció que el objetivo de la educación en el país es "contribuir al desarrollo integral del individuo para que ejerza plenamente sus capacidades humanas".

De la misma manera, la Ley 115 de 1994 o Ley General de Educación definió que la educación es un proceso de formación permanente, personal, cultural y social que se fundamenta en una concepción integral de la persona humana, de su dignidad, de sus derechos y de sus deberes. Tampoco en su contenido se hace explícito el término o el tema de la tutoría.

En Colombia, la tutoría se ha venido implementando especialmente en el ámbito universitario, sobre lo cual se pueden retomar algunas de esas experiencias.

Una de las prioridades actuales del sistema educativo colombiano es cambiar los paradigmas de la educación por un sistema de formación integral de calidad que desarrolle valores, actitudes, habilidades, destrezas y aprendizajes significativos.

En el SENA. El Acuerdo 12 de 1985 por medio del cual se establecen los lineamientos fundamentales de la política Técnico-Pedagógica del SENA y se fijan las directrices para su gestión con miras a lograr y conservar la Unidad Técnica en la Entidad, define el término tutoría como "una estrategia para facilitar a los docentes la orientación de acciones de formación a distancia, previa formación de los mismos". Así, el aprendizaje individual es apoyado por la tutoría que 
orienta al aprendiz a lo largo del proceso de formación, ya sea por correspondencia, telefónicamente, por medio de audiovisuales y grabaciones u otros medios. En este recorrido, a la formación a distancia, se le incorpora luego una fase presencial, la cual busca que los aprendices se reúnan para discutir problemas comunes, reforzar aprendizajes, realizar prácticas y organizar formas de aplicación colectiva y productiva de los aprendizajes logrados con el apoyo y orientación de un tutor. En 2003 surgió la formación virtual como aporte y alternativa a la formación presencial y a la educación a distancia. Aparece un nuevo rol de tutor con la función de orientar los programas y actividades en la plataforma virtual del SENA.

En 2004 la entidad adopta formalmente el enfoque por competencias y el aprendizaje por proyectos en su estrategia formativa; para ello se basa en las cuatro fuentes de conocimiento (el entorno, el trabajo colaborativo, las tic, instructor-tutor), redefiniendo el rol del instructor como tutor, orientador, facilitador y acompañante del aprendiz, no solo en lo académico sino también en la parte personal. De igual manera, el Plan Estratégico del SENA 2011 - 2014 plantea la formación como mejores seres humanos y mejores ciudadanos como una de las principales estrategias para mejorar la integralidad de la formación del aprendiz, lo cual lleva implícita la práctica tutorial.

\section{Técnicas de recolección de información}

Con el fin de reforzar la información cualitativa, el equipo investigador se apoyó en información de tipo cuantitativo. Los instrumentos utilizados fueron:

\section{Encuesta semiestruturada}

Es un procedimiento para la recolección de datos facilitados por los encuestados a través de cuestionarios, según un diseño previamente establecido. Su función primordial es la estandarización del proceso de recogida de datos por medio de un cuestionario con preguntas que buscan abarcar las dimensiones del tema a investigar. La encuesta semiestructurada se aplicó a nueve instructores y a 90 aprendices, cuestionario de 24 preguntas cerradas con categoría de respuesta tipo Likert (5 - Siempre, 4 - Muchas veces, 3 - Algunas veces, 2 - Pocas veces y 1 - Nunca).

\section{Grupo focal}

Es una entrevista grupal abierta y estructurada que tiene como propósito registrar la forma como unos participantes que son previamente seleccionados por los investigadores, discuten desde su experiencia, un tema o evento que es objeto de investigación. Es una participación dirigida y consciente que permite llegar a unas conclusiones. Se realizaron dos grupos focales, el primero con cuatro instructores tutores que atienden la formación y otro con seis aprendices de la Tecnología en Gestión de Mercados.

\section{Revisión documental}

Es el proceso que permite recopilar el material, registrar, analizar, clasificar la información que surge de diversas fuentes, para obtener los elementos que den respuesta a la pregunta de investigación; para ello se selecciona lo que pueda contribuir a ese propósito y por último se elabora el esquema conceptual que define la forma como se presentarán las ideas con base en la pregunta que motivó la investigación. Se hizo rastreo con el fin de ubicar, seleccionar y consultar las fuentes y los documentos que dan cuenta de la práctica tutorial en los contextos internacional, nacional y en el SENA.

\section{Resultados y discusión}

\section{- Componente cognitivo}

\section{Habilidad de análisis}

- El $36 \%$ de los aprendices encuestados indica que sus instructores: A Veces, Pocas Veces o Nunca les ayudan a analizar los contenidos de las guías de aprendizaje.

- El $72 \%$ de los aprendices afirma que: Algunas Veces, Casi Nunca o Nunca el instructor se interesa porque "reflexionemos sobre el contenido de las guías”.

- El 39 \% de los aprendices respondió Siempre o Muchas Veces al enunciado "El instructor no se interesa porque los aprendices logremos la comprensión de las guías".

Situaciones como esta dan cuenta de la necesidad de fortalecer las habilidades cognitivas de los aprendices, 


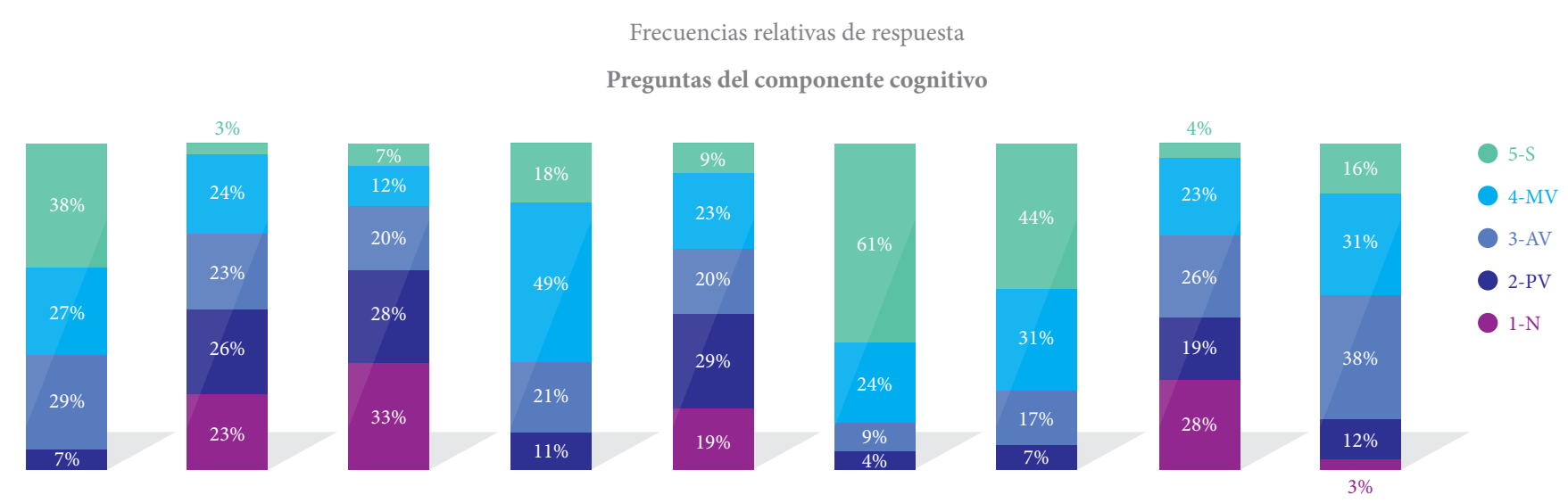

Figura 1. Frecuencias relativas de respuesta a las preguntas del componente cognitivo.

implementando estrategias que les permitan apropiarse del contenido de las guías y puedan profundizar en el análisis.

Pasar inadvertida la posibilidad de comprender equivale, si se quiere, a impedir aprender. De la mano de la reflexión va la indagación; la comprensión implica la habilidad de pensar sobre lo que se sabe para indagar sobre lo deseable, de aquello desconocido que puede ser aprehensible. Para Clavijo (2010:26), "la comprensión es la capacidad de usar conocimientos, conceptos y habilidades para iluminar nuevos problemas o temas no previstos". Así mismo, plantea que "la comprensión se presenta cuando una persona puede pensar y actuar con flexibilidad a partir de lo que sabe".

Lo anterior se corrobora con las opiniones que los aprendices manifestaron en el grupo focal: "Los profesores empiezan con la batería bajita y se van viendo alcanzados con el mismo proceso..., entonces empezamos bacano, relajaditos estudiando todo chévere cuando a última hora, todo a las carreras, porque faltan varios temas para terminar las guías". "Hacemos los entregables, no por aprender sino por cumplir, porque como no tenemos el conocimiento suficiente". "Por lo general, nosotros como estudiantes somos muy despistados y no entendemos y necesitamos al profesor y lo llamamos, y solo nos atiende en horario de clase". "Pero si uno no entiende desde el principio, así sea que le manden tres mil planes de mejoramiento, si el profesor no explica bien, no lo vamos a entender".

Vale la pena resaltar la anotación que hace Díaz, F. (citada por Dávila S. 2000), quien hace una apreciación que va más allá de la labor de facilitador por parte del docente; él hace referencia a la labor de la mediación, así:

La función del trabajo docente no puede reducirse ni a la de simple transmisor de la información, ni a la de facilitador del aprendizaje. Antes bien, el docente se constituye en un mediador en el encuentro del estudiante con el conocimiento. En esta mediación el profesor orienta y guía la actividad mental constructiva de sus alumnos, a quienes proporciona ayuda pedagógica ajustada a su competencia. (Díaz 2000).

\section{Habilidad de argumentación}

- El $32 \%$ de los aprendices considera que Algunas Veces o Pocas Veces en los ambientes de aprendizaje, los instructores les enseñan a diferenciar las opiniones personales de los contenidos de las guías.

- El $68 \%$ de los aprendices responde que Algunas Veces, Pocas Veces o Nunca al enunciado "El instructor promueve discusiones sobre los contenidos de las guías".

- El $85 \%$ de los aprendices manifiesta que Siempre o Muchas Veces el instructor no tiene en cuenta sus opiniones frente a los temas de las guías de aprendizaje.

Si bien es cierto, que estas habilidades se trabajan por separado, todas ellas se correlacionan entre sí, es decir, que cuando falta una, se afecta el desarrollo de las otras, tal como se indicó anteriormente cuando se trató la reflexión como habilidad de pensamiento, por 
medio de la cual se hace consciente el aprendizaje y al mismo tiempo permite evaluar y cuestionar la nueva información, someterla a la crítica, pedir razones y sustentar con rigor lo que se expone; separar la opinión de lo textual.

La falta de discusión sobre el tema objeto de estudio, que en este caso son las guías de aprendizaje, son oportunidades desaprovechadas por los instructores para potenciar en sus aprendices los componentes cognitivo, social y emocional. Es precisamente en el diálogo sobre los contenidos de las guías el escenario propicio para promover interacciones efectivas entre instructor y aprendices y entre estos y sus iguales.

Retomando a Cuenca (1995, pp. 23-40), "La argumentación es una forma de interacción comunicativa particular en la que docentes y alumnos confrontan sus saberes, sus opiniones sobre un tema determinado"; por su parte Camp \& Dolz, (1995), consideran que el discurso argumentativo le permite al individuo resolver, por medio de la palabra, las situaciones que surjan en su entorno, para sustentar sus ideas, asumir posiciones críticas frente a otros y dar soluciones a posibles conflictos.

\section{Habilidad de indagación}

- El 75 \% de los aprendices afirma que Siempre o Muchas Veces los instructores los motivan para que consulten en otras fuentes los temas de las guías de aprendizaje.

- El73\% delos aprendices manifiestan que Algunas Veces, Pocas Veces o Nunca los instructores los motivan para que formulen preguntas sobre los contenidos de las guías de aprendizaje.

- El $47 \%$ de los aprendices responde Siempre o Casi Siempre el instructor prefiere de ellos las respuestas, más que las preguntas.

Resulta contradictorio que el instructor motive al aprendiz a consultar otras fuentes de información y que estas no sean confrontadas con el contenido de las guías en el proceso de formación, ya que con ello se pierde la oportunidad de fortalecer el aprendizaje y el surgimiento de nuevos interrogantes.
Los resultados muestran como algunos instructores se consideran protagonistas del proceso formativo, lo que conlleva a un distanciamiento con los aprendices, situación que es más preocupante cuando estos manifiestan que a los instructores les da temor que les hagan preguntas por considerarlas una amenaza o un riesgo a su credibilidad.

Ausubel et al., (1976) ya se había referido a la necesidad que existe de que el sujeto esté dispuesto a ese aprendizaje significativo para que este se haga efectivo. Si esa condición no se da, el aprendizaje se quedará simplemente en algo mecánico y reproductivo. Por eso adquiere suma importancia que asuma el papel del docente en lo que se refiere a la creación de un ambiente apropiado, el cual motive al aprendiz para que se disponga y propicie su proceso de aprendizaje significativo.

Lo anterior es corroborado por los aprendices desde el grupo focal, quienes afirmaron: "Francamente no me siento motivada para investigar". "Lo que lo motiva a uno a investigar son los sermones del profe: es que le está yendo muy mal, usted se va a ir de acá, se echa solito". "Y uno dice: me voy a tener que poner las pilas porque aquí me van es a sacar". "Es el instructor el que nos debe motivar a investigar". "Yo investigo porque me gusta, porque estoy estudiando y porque sé que lo tengo que hacer para aprender; es más por iniciativa personal".

Estudiosos de lo cognitivo como Piaget (1974), dice:

El principal objetivo de la educación consiste en formar personas que sean capaces de hacer cosas nuevas y no simplemente de repetir lo que en otras generaciones han realizado. Se necesitan hombres que sean creadores, que estén pletóricos de inventiva y que sean capaces de descubrir algo original. El segundo objetivo de la pedagogía consiste en formar mentes críticas, ávidas de licor de la verdad y que no estén dispuestas a aceptar gratuitamente todo lo que se les ofrece. El gran peligro que se cierne sobre nuestras cabezas consiste hoy en día en los tópicos, en las frases hechas y que repiten como papagayos las masas. Tenemos que ser capaces de resistir a esta presión, de criticar y de distinguir entre lo que es verdad y los que es mera opinión. Necesitamos para ello alumnos activos, capaces de aprender por sí mismos, en parte gracias a su actividad espontánea y en parte también a través de los datos que les brindemos; alumnos que aprendan rápidamente a distinguir entre lo que es verídico y lo que es gratuito. 
Es claro que el instructor ya no es la fuente principal del conocimiento, ni determina las generalizaciones que se van a aprender, ni sanciona sus equivocaciones en la realización de las tareas, sino que su papel es de mediador y orientador de sus aprendices para ayudarlos a descubrir sus aciertos y aprender de sus errores a través de una serie de preguntas que iluminan el camino del aprendizaje.

Al respecto Vygotsky (1978) plantea que el alumno encuentra posibilidades de aprendizaje cuando descubra experiencias de conflicto cognitivo, o lo que es lo mismo, cuando pueda contemplar las múltiples perspectivas de un mismo fenómeno, suceso o principio. Ya se había mencionado de Vygotsky (1978:
12) la Zona de Desarrollo Próximo que definió como aquellas funciones que aún no han madurado en el individuo, pero que están en proceso de maduración. Esto quiere decir, que "la enseñanza solo es eficaz cuando se sitúa dentro de esa zona de desarrollo, ya que si se sitúa por encima, se ahoga el alumno y no aprende; y si se coloca por debajo, se aburre".

En síntesis, el componente cognitivo dentro de la investigación "Las prácticas tutoriales y la formación integral del aprendiz del SENA" muestra posibilidades de mejora en lo que respecta a la forma como el instructor promueve en el aprendiz el desarrollo de las habilidades propias de este componente.

Frecuencias relativas de respuesta

Preguntas del componente emocional

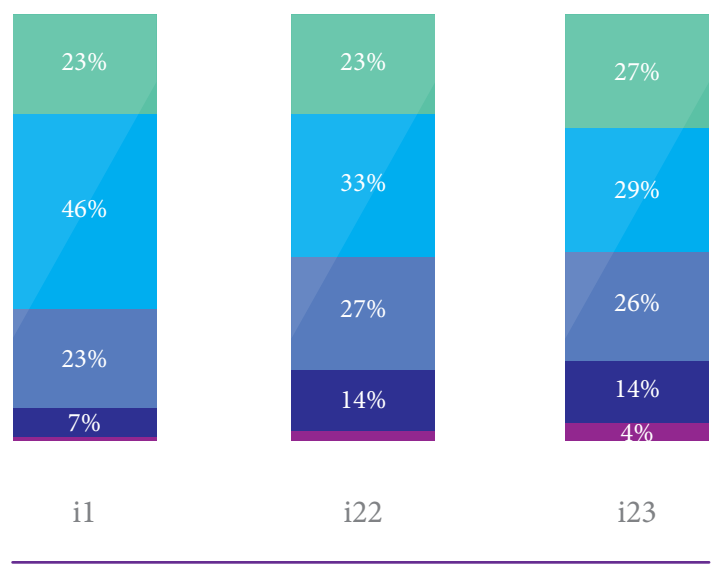

Empatía

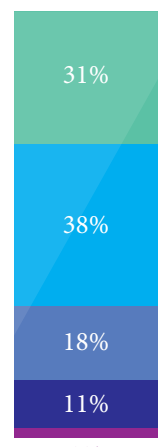

$2 \%$

i13

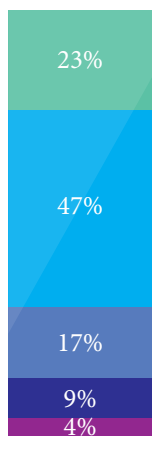

i16

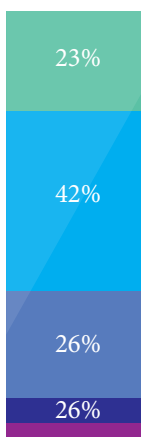

$3 \%$

i19-
Tolerancia

Figura 2. Frecuencias relativas de respuesta a las preguntas del componente emocional.

\section{- Componente emocional}

\section{Habilidad de empatía}

- El $30 \%$ de los aprendices manifiesta que Pocas Veces o Nunca, el instructor se muestra solidario ante las dificultades del aprendiz respetando las diferencias.

- El $44 \%$ de los aprendices responde que Algunas Veces, Pocas Veces o Nunca, el instructor muestra consideración ante las necesidades académicas o personales que afectan su permanencia en la formación.
- El $44 \%$ de los aprendices afirma que Algunas Veces, Pocas Veces o Nunca el instructor, ante situaciones de conflicto, se preocupa por escuchar atentamente y por igual a las partes implicadas.

Resultados como estos guardan consistencia con las falencias detectadas en el componente cognitivo, e incluso, con el componente social, pues a mayor distanciamiento entre instructores y aprendices, menor será la sensibilidad entre ambos, lo que lleva a un deterioro de las relaciones. 
Estos componentes se correlacionan de tal manera que de las habilidades que se tengan o no de un componente, depende la activación de habilidades de otro componente. Por lo visto, la afectación de las habilidades cognitivas opaca las habilidades de orden emocional y viceversa.

Frente a estos aspectos, en el grupo focal los aprendices manifestaron: "Algunos instructores son muy llevados de su parecer, es lo que ellos digan y así tiene que ser". "Pero si el instructor no muestra actitud y ganas no le pueden brindar nada al aprendiz y por ese lado la mayoría de veces no funciona y muchos aprendices terminan retirándose".

Según Roche (1995), al analizar el concepto de empatía, se distinguen dos componentes: el cognitivo y el afectivo. En efecto, en la empatía se hallan implicados procesos racionales y emocionales, ya que nos permite comprender a los demás, generalmente en lo que se refiere a sus estados de ánimo. La empatía, por tanto, es la capacidad para ponerse en el lugar del otro. Gracias a la empatía nos adentramos en la realidad personal de los demás.

\section{Habilidad de tolerancia}

- El $31 \%$ de los aprendices manifiesta que A Veces, Pocas Veces o Nunca el instructor mantiene la calma ante situaciones conflictivas en el ambiente de aprendizaje.

- El $30 \%$ de los aprendices afirma que Algunas Veces, Pocas Veces o Nunca, el instructor asume nuestros errores como oportunidad para volver a explicar el tema con mayor claridad.

- El 65 \% de los aprendices responde que Siempre o Casi Siempre el instructor se enoja con facilidad ante nuestras dificultades académicas o comportamentales.

Es evidente que mientras las demandas de orden emocional aumentan en el contexto familiar, social, académico y laboral, apenas ahora se empieza a considerar su importancia desde la educación. Es muy significativo saber que en el SENA esta problemática es reconocida por los instructores aunque algunos consideran que esto es responsabilidad de otras disciplinas, según lo manifestaron en el grupo focal.
Frente a este aspecto, en el grupo focal los aprendices afirmaron: "A los instructores les da mucha rabia que uno les lleve la contraria". "Muchas veces uno sabe que tiene la razón y le toca a uno revaluar porque es el instructor y entonces también eso es como un desprestigio para uno". "No queremos entrar en conflicto con el profe, por eso uno hasta se abstiene de pedir más explicaciones y uno se queda ahí, pero por lo general sí se irritan mucho". "Pero cuando las cosas no funcionan, simplemente nos dicen retírense de la formación, que esto no es lo suyo".

$\mathrm{Al}$ respecto, las competencias emocionales se consideran un aspecto importante de las habilidades de empleabilidad. En el mundo laboral se acepta que la productividad depende de una fuerza de trabajo que sea emocionalmente competente (Elías et al., 1997: 6; Goleman, Boyatzis y McKee, 2002). En este mismo plano, Rafael Flórez (1989), advierte que la disposición del docente para promover el gusto, el interés y la forma en cómo se apropia el conocimiento científico es uno de los factores que más influye en el aprendizaje de los estudiantes. Aquí emerge otro factor importante que bien puede complementar lo que hasta aquí se viene tratando, y se refiere precisamente al gusto y el interés que promueve el instructor sobre el objeto de aprendizaje, diferente a intimidar o hacer difícil lo que puede ser aprendido, tal como lo expresaron los aprendices en el grupo focal.

\section{- Componente social}

\section{Habilidad de acompañamiento}

- El $43 \%$ de los aprendices manifestaron que Algunas Veces, Pocas Veces o Nunca el instructor atiende oportunamente nuestras solicitudes frente a cualquier dificultad académica $\mathrm{o}$ personal.

- El 36 \% de los aprendices responde que Pocas Veces o Nunca el instructor les da seguridad y confianza para que expresen sus ideas frente al desarrollo de las guías de aprendizaje.

- El 50 \% de los aprendices responde que Algunas Veces, Pocas Veces o Nunca el instructor le da importancia al aprendizaje colaborativo entre aprendices. 


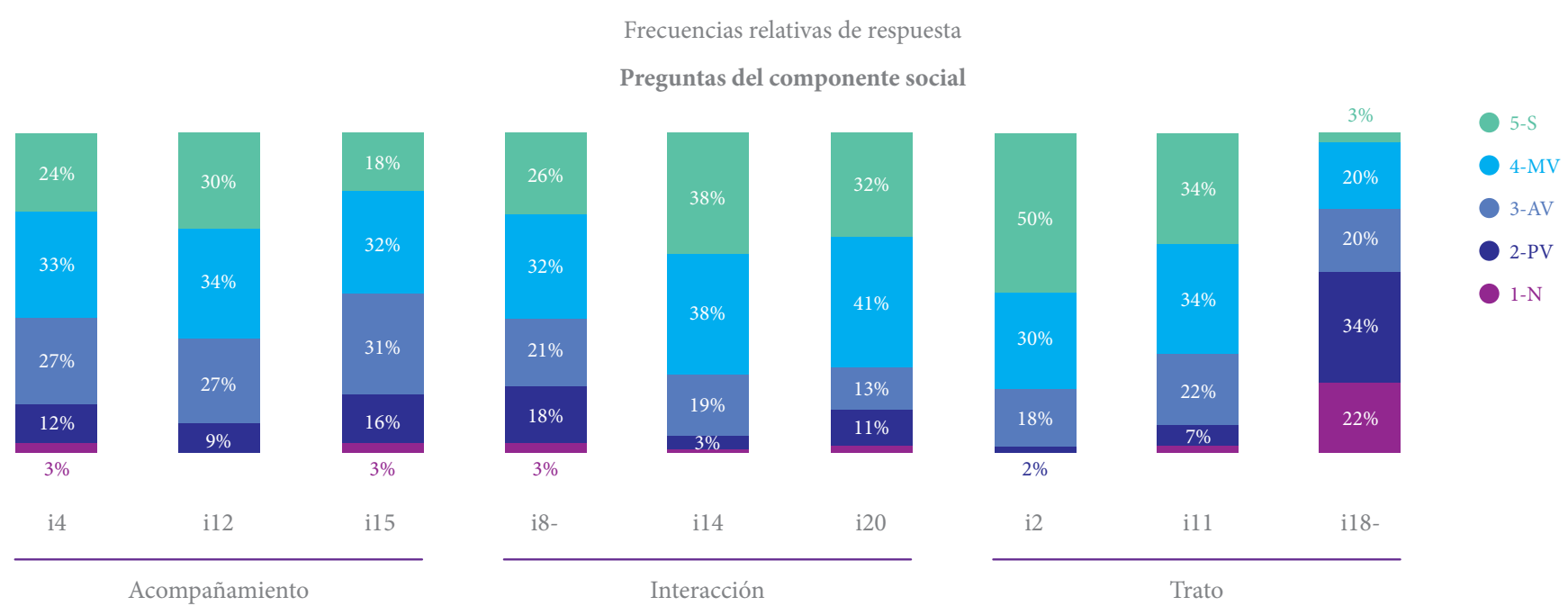

Figura 3. Frecuencias relativas de respuesta a las preguntas del componente social.

Esta apreciación está relacionada con el análisis realizado a los componentes cognitivo y emocional, donde nuevamente se refleja la necesidad de promover una mayor interacción y mediación entre el instructor y sus aprendices y desde luego, de sus demandas cognitivas, emocionales y sociales.

Al respecto, estas fueron las percepciones de los aprendices que participaron en el grupo focal: "Para nosotros, como aprendices, es muy difícil trabajar en equipo". "Nos toca manejar temperamentos muy distintos, personalidades muy distintas, hay unos que sí llegamos con ganas de estudiar, hay otros que vienen con ganas de calentar el puesto". "Nos toca trabajar por los otros y orientarlos para que medio respondan porque tampoco es que respondan del todo". "El instructor se limita a responder: usted tuvo su tiempo para conocer a las personas con quien iba a trabajar, ya eso es problema suyo, yo cumplo con decirles quiénes serán sus compañeros".

Bruner (1995), en su obra "Actos de significado. Más allá de la revolución cognitiva”, sostiene que el objetivo es orientar al sujeto en el camino hacia la cultura, para comprenderla en sus complejidades y contradicciones; el propósito es cultivar las creencias, habilidades y sentimientos para que el sujeto explique e interprete el mundo y logre así modificar la sociedad de la que hace parte.

\section{Habilidad de interacción}

- El $79 \%$ de los aprendices afirma que Siempre, Casi Siempre o Algunas Veces el instructor prefiere que los aprendices trabajemos más de manera individual que en grupo.

- El $76 \%$ de los aprendices manifiesta que Siempre $o$ Casi Siempre el instructor se interesa porque los aprendices resolvamos las guías en grupo dentro del ambiente de aprendizaje.

- El 73 \% de los aprendices responde que Siempre $o$ Casi Siempre el instructor promueve relaciones de entendimiento entre los aprendices.

Es reiterativa la negación a trabajar con los otros, lo que revela la necesidad de un mejor entendimiento entre instructores y aprendices, para lograr que estos últimos reconozcan la importancia del trabajo en equipo.

Frente a este aspecto, en el grupo focal los aprendices se pronunciaron así: "De pronto yo creería que la estrategia que ustedes deben utilizar es la convivencia, mas no el trabajo en equipo para hacer la actividad". "Se requiere fortalecer más la convivencia entre los equipos, que es lo realmente importante, porque a la hora de la verdad cuando va a trabajar se trabaja independiente". "Yo creo que más que todo el error está en el acompañamiento". "Para mí es muy tedioso 
trabajar en grupo, y más, cuando me toca en un grupo con personas que no quiero".

Mélich (1994), uno de los estudiosos del acto educativo, específicamente en la orientación del proceso educativo, considera que en las interacciones pedagógicas que cobran vida en este, radica el secreto de la orientación del proyecto formativo.

Estos resultados muestran la necesidad de fortalecer en los instructores la formación en el aprendizaje por proyectos, pues ellos mismos reconocen sus falencias en esta competencia para hacer acompañamiento más efectivo. No obstante se resalta su interés cuando expresan que hacen un diagnóstico al iniciar la formación, pero no se hace un adecuado seguimiento. De otro lado reconocen que los aprendices no llegan de la educación media con muchos vacíos, y en ocasiones asumen expresiones que indican que ya no hay nada por hacer con algunos: "A ellos los mataron desde el colegio, les metieron un chip muy raro, les metieron otra cosa”. En estas últimas afirmaciones se muestra la necesidad de revisar las competencias que trae el aprendiz desde la educación media y la conveniencia de que algunos instructores reciban formación en los componentes cognitivo, social y emocional.

\section{Habilidad en el trato}

- El $80 \%$ de los aprendices manifiestan que Siempre o Casi Siempre el instructor se dirige a los aprendices con respeto y cordialidad, dentro y fuera del ambiente de aprendizaje.

- El 68 \% de los aprendices afirman que Siempre y Casi Siempre el instructor exige respeto en las relaciones entre compañeros aprendices, dentro y fuera del ambiente de aprendizaje.

Estos resultados fueron corroborados en las expresiones de los aprendices que participaron en el grupo focal: "Los profesores se dedican a hacer lo de ellos". "Uno les dice: profe, hay un conflicto entre estas personas, y él no se preocupa hasta que el conflicto estalla". "El profe se queda relajado hasta que no ve la gravedad de las cosas". Manifestaciones como estas, muestran la inconsistencia con los resultados ya señalados (el $80 \%$ de los instructores se dirigen con respeto a los aprendices y el $68 \%$ afirma que el instructor exige respeto en las relaciones entre aprendices).
Mélich (2002) plantea que la relación pedagógica, a diferencia de otras, es una relación intencional en la que se propician diferentes modos de aprender; ayuda al estudiante en la adquisición de recursos para desempeñarse en diferentes espacios y situaciones . Sin embargo, no es suficiente la simple cercanía con el objeto a conocer. Ya Oliveira (1996) había advertido que eso no garantiza el aprendizaje, así como el hecho de interactuar en un ambiente formador tampoco garantiza que se promueva el desarrollo del aprendiz desde la óptica cultural. Así mismo, Pérez Gómez y Soto (2009), afirman que el aprendizaje relevante y duradero es ante todo el resultado de la participación del individuo en prácticas sociales, por ser miembro de una comunidad social, y que las habilidades, actitudes, valores y conocimientos tienen lugar como parte de un proceso de familiaridad con formas de ser, pensar, sentir y ver del grupo, y el entorno social en el que se desenvuelve.

Una de las mayores preocupaciones del SENA, si quiere rescatar la integralidad de la formación, es promover el desarrollo de las competencias socioafectivas, tal como lo plantea Shapiro (1997).

En síntesis, el componente social es uno de los mejor valorados por los aprendices, especialmente en el buen trato que reciben por parte de sus instructores, fortaleza que si se quiere, puede servir de base para la potenciación de las demás habilidades sociales, cognitivas y emociones que deben desarrollar (Tabla $1)$.

Si se aprobara la calificación con un promedio de 3,0 , el resultado para el componente cognitivo fue de 3,1 . Esto explica la necesidad de que los instructores mejoren las habilidades de análisis, argumentación e indagación y las promuevan entre sus aprendices. Los resultados muestran que la argumentación fue reprobada con un 2,7; la indagación está en el límite con un 3,1 y el análisis superó levemente el promedio con un 3.4. Siguiendo la comparación inicial, tanto las que están por debajo de 3 como las que lo superan (3.4), evidencian la necesidad de hacer una intervención inmediata, en aras de mejorar esa competencia en los instructores tutores del Centro de Comercio del SENA - Regional Antioquia.

Con respecto al componente emocional, este tuvo un promedio de 3,5, y la habilidad de mayores 
Tabla 1. Análisis de los resultados por componente y habilidad asociada.

\begin{tabular}{ccccccccc} 
& \multicolumn{2}{c}{$\begin{array}{c}\text { Componente } \\
\text { cognitivo }\end{array}$} & \multicolumn{2}{c}{$\begin{array}{c}\text { Componente } \\
\text { emocional }\end{array}$} & \multicolumn{2}{c}{ Componente social } \\
\hline $\begin{array}{c}\text { Indi- } \\
\text { cador }\end{array}$ & $\begin{array}{c}\text { Aná- } \\
\text { lisis }\end{array}$ & $\begin{array}{c}\text { Argumen- } \\
\text { tación }\end{array}$ & $\begin{array}{c}\text { Indaga- } \\
\text { ción }\end{array}$ & Empatía & $\begin{array}{c}\text { Tole- } \\
\text { rancia }\end{array}$ & $\begin{array}{c}\text { Acompa- } \\
\text { namiento }\end{array}$ & $\begin{array}{c}\text { Intera- } \\
\text { cción }\end{array}$ & Trato \\
\hline $25 \%$ & 3,0 & 2,3 & 2,7 & 3,0 & 3,0 & 3,1 & 3,0 & 3,3 \\
$50 \%$ & 3,3 & 2,7 & 3,0 & 3,7 & 3,3 & 3,7 & 3,3 & 4,0 \\
Promedio & 3,4 & 2,7 & 3,1 & 3,7 & 3,3 & 3,6 & 3,5 & 3,9 \\
$75 \%$ & 4,0 & 3,0 & 3,3 & 4,3 & 3,7 & 4,3 & 3,7 & 4,3 \\
Desviación & 0,6 & 0,6 & 0,6 & 0,8 & 0,6 & 0,8 & 0,6 & 0,7 \\
\hline
\end{tabular}

Fuente: Elaboración propia con base en los resultados de la encuesta realizada en 2012.

oportunidades de mejora fue la tolerancia, que obtuvo una calificación promedio de 3,3. Lo anterior, comparado con un puntaje de 5 como referente, advierte la debilidad de este componente en la formación de los aprendices del SENA.

Al componente social le correspondió un promedio de 3,7. La habilidad de mayor demanda en los instructores es la interacción, la cual obtuvo una calificación promedio de 3,5; y el acompañamiento obtuvo una calificación de 3,6. La mayor fortaleza se percibió en el trato que dan a sus aprendices con una calificación promedio de 3,9.

Sin embargo, para los tres componentes evaluados (Cognitivo, Emocional y Social) se obtuvo una calificación promedio de 3,4. Aquí aparece uno de los principales hallazgos de esta investigación, con el cual puede explicarse en parte la razón por la cual el SENA hoy no logra dar respuesta a uno de sus mayores retos: la calidad en la formación para el trabajo, explicado por deficiencias en la integralidad de la formación.

\section{Conclusiones}

- La práctica tutorial del Instructor de Tecnología en Gestión de Mercados del Centro de Comercio del SENA - Regional Antioquia, no es suficiente para promover en sus aprendices las habilidades en los componentes social, cognitivo y emocional. Los resultados muestran que el componente cognitivo, especialmente en las habilidades de argumentación e indagación, es el que mayor vacío presenta en la formación integral del aprendiz del SENA y está estrechamente ligado a los componentes social y emocional.

- Los instructores que participaron en el grupo focal reconocieron la dificultad que tienen para vincular a su práctica docente los componentes cognitivo, social y emocional de sus aprendices, argumentando que no poseen la formación necesaria y que esto ha sido considerado competencia de otras instancias del SENA.

- Los aprendices participantes en la investigación reclaman con vehemencia un mayor acompañamiento por parte del instructor tutor, dado que su formación integral implica no solo la adquisición de conocimientos técnicos, sino también la internalización de valores, actitudes y formas de comportamiento que contribuyan al mejoramiento de sus condiciones sociales y laborales.

- Los resultados de la investigación le permitirán al SENA implementar estrategias para entregar a la sociedad aprendices con las competencias técnicas y actitudinales para un óptimo desempeño en el mercado laboral.

- Ajustar los procedimientos que tiene el SENA para la selección de aspirantes a instructor, con el fin de 
garantizar en estos profesionales las competencias, tanto del área técnica como las asociadas a los componentes social, cognitivo y emocional, requeridas en la formación profesional integral.
- Revisar y ajustar el actual modelo pedagógico institucional, con el objeto de recuperar el protagonismo del aprendiz como eje central de la formación profesional integral del SENA.

ANEXO 1. Encuesta realizada al instructor-tutor.

\begin{tabular}{|c|c|c|c|c|c|c|}
\hline Nro. & Usted como instructor-tutor & Siempre & $\begin{array}{l}\text { Muchas } \\
\text { veces }\end{array}$ & $\begin{array}{l}\text { Algunas } \\
\text { veces }\end{array}$ & $\begin{array}{l}\text { Pocas } \\
\text { veces }\end{array}$ & Nunca \\
\hline 1 & $\begin{array}{l}\text { Se muestra solidario ante las dificultades de los aprendices } \\
\text { respetando las diferencias. }\end{array}$ & & & & & \\
\hline 2 & $\begin{array}{l}\text { Se dirige a sus aprendices con respeto y cordialidad dentro y } \\
\text { fuera del ambiente de aprendizaje. }\end{array}$ & & & & & \\
\hline 3 & $\begin{array}{l}\text { Le ayuda a sus aprendices a analizar los contenidos de las guías } \\
\text { de aprendizaje. }\end{array}$ & & & & & \\
\hline 4 & $\begin{array}{l}\text { Atiende oportunamente las solicitudes de sus aprendices frente a } \\
\text { cualquier dificultad académica o personal. }\end{array}$ & & & & & \\
\hline 5 & $\begin{array}{l}\text { Los incentiva para que consulten en otras fuentes los temas de las } \\
\text { guías de aprendizaje. }\end{array}$ & & & & & \\
\hline 6 & $\begin{array}{l}\text { Les enseña a diferenciar las opiniones personales de los } \\
\text { contenidos de las guías de aprendizaje. }\end{array}$ & & & & & \\
\hline 7 & $\begin{array}{l}\text { Prefiere que sus aprendices trabajen más de manera individual } \\
\text { que en grupo. }\end{array}$ & & & & & \\
\hline 8 & $\begin{array}{c}\text { Promueve las discusiones sobre los contenidos de las guías de } \\
\text { aprendizaje. }\end{array}$ & & & & & \\
\hline 9 & $\begin{array}{c}\text { No tiene en cuenta las opiniones de sus aprendices frente al tema } \\
\text { de la guía de aprendizaje. }\end{array}$ & & & & & \\
\hline 10 & $\begin{array}{l}\text { Exige respeto en las relaciones entre compañeros dentro y fuera } \\
\text { del ambiente de aprendizaje. }\end{array}$ & & & & & \\
\hline 11 & $\begin{array}{l}\text { Les da seguridad y confianza a sus aprendices para que expresen } \\
\text { sus ideas frente al desarrollo de las guías de aprendizaje. }\end{array}$ & & & & & \\
\hline 12 & $\begin{array}{c}\text { Mantiene la calma ante situaciones conflictivas en el ambiente de } \\
\text { aprendizaje. }\end{array}$ & & & & & \\
\hline 13 & $\begin{array}{l}\text { Se interesa por que sus aprendices resuelvan las guías en grupo } \\
\text { dentro del ambiente de aprendizaje. }\end{array}$ & & & & & \\
\hline 14 & Da importancia al aprendizaje colaborativo entre aprendices. & & & & & \\
\hline 15 & $\begin{array}{l}\text { Asume los errores de sus aprendices como oportunidad para } \\
\text { volver a explicar el tema con mayor claridad. }\end{array}$ & & & & & \\
\hline 16 & $\begin{array}{l}\text { Los motiva para que formulen preguntas sobre los contenidos de } \\
\text { las guías de aprendizaje. }\end{array}$ & & & & & \\
\hline 17 & $\begin{array}{l}\text { Es indiferente frente al trato irrespetuoso de algunos aprendices } \\
\text { dentro y fuera del ambiente de aprendizaje. }\end{array}$ & & & & & \\
\hline 18 & $\begin{array}{l}\text { Se enoja con facilidad ante las dificultades académicas o } \\
\text { comportamentales del aprendiz. }\end{array}$ & & & & & \\
\hline 19 & Promueve relaciones de entendimiento entre los aprendices. & & & & & \\
\hline 20 & $\begin{array}{l}\text { Se interesa porque los aprendices reflexionen sobre el contenido } \\
\text { de las guías de aprendizaje. }\end{array}$ & & & & & \\
\hline 21 & Prefiere de sus aprendices las respuestas más que las preguntas. & & & & & \\
\hline 22 & $\begin{array}{l}\text { Muestra consideración frente a las necesidades académicas o } \\
\text { personales que afectan la continuidad de sus aprendices en el SENA. }\end{array}$ & & & & & \\
\hline 23 & $\begin{array}{l}\text { Frente a situaciones de conflictos se preocupa por escuchar } \\
\text { atentamente y por igual a cada una de las partes implicadas. }\end{array}$ & & & & & \\
\hline 24 & $\begin{array}{c}\text { No se interesa por que sus aprendices logren la comprensión de las } \\
\text { guías de aprendizaje. }\end{array}$ & & & & & \\
\hline
\end{tabular}




\section{Referencias}

Ausubel, D. et al. Psicología Cognitiva. (1976). Un punto de vista cognoscitivo. México.

Bruner, J. (1991). Actos de significado. Más allá de la revolución cognitiva. pp.153. Madrid, España: Ed. Alianza.

Clavijo, A. (2010). La enseñanza para la comprensión: una forma de pensar desde la complejidad. Revista "Entre Comillas", 13, 26.

Cuenca, M.J. (1995). Mecanismos lingüísticos y discursivos de la argumentación. Comunicación, Lenguaje y Educación, 25, 23-40

Dávila, S (2000). El aprendizaje Significativo. Revista Digital Contexto Educativo, No. 9, julio de 2000. Dávila, S (2000). El aprendizaje Significativo. Revista Digital Contexto Educativo, No. 9, julio de 2000.

De Oliveira, M.K. (1996). Pensar la educación: Las contribuciones de Vygotsky. pág. 52

Elías, M., et al. (1997). Promoting social and emotional learning. Guidelines for Educators. Alexandria, Virginia: ASCD (Association for Supervision and Curriculum Development).

Flórez R. (1999) Evaluación Pedagógica y Cognición. McGrawHill.
Goleman, D., Boyatzis, R., McKee, A. (2002). EL Líder Resonante crea más. El poder de la inteligencia emocional. Barcelona: Plaza y Janés.

Mélich, J. (1994). Del extraño al cómplice. la educación en la vida cotidiana. Barcelona, España: Anthropos.

Monroy, L. (1994). La estructura del ser humano como fundamento de la educación en lo superior y para lo superior: bases para un diseño curricular aplicable a la formación integral universitaria. (p. 148). Cali, Colombia: Universidad del Valle.

Pérez Gómez, A.I. y Soto Gómez, E. (2009). "Competencias $y$ contextos escolares. Implicaciones mutuas". Organización y Gestión Educativa, 2, 17-22.

Prawda, H. A. (2008). "Mediación escolar sin mediadores". 1era. Ed., Buenos Aires: Bonum. pp. 12

Roche R. (1995): Psicología y educación para la prosocialidad, Barcelona, Universidad Autónoma. P. 141

Shapiro, L. (1997). La inteligencia emocional de los niños. Grupo Zeta, Bilbao, España.

Timudez, O. (2010). Escuelas complementarias. Revista Ciencias de la Educación, 20(35), 267-276. 